\title{
P-wave Duration and Dispersion in Lone Obesity
}

\author{
Mehmet Cosgun, Isa Sincer, Mehmet Inanir, Emrah Erdal, Asli Kurtar Mansiroglu and Yilmaz Gunes \\ Department of Cardiology, Bolu Abant Izzet Baysal University Hospital, Bolu, Turkey
}

\begin{abstract}
Objective: To assess P-wave duration and dispersion (PD) in morbidly obese young subjects who do not have co-associated atrial fibrillation (AF) risk factors, such as hypertension, diabetes, atrial enlargement and diastolic dysfunction.

Study Design: An observational cross-sectional study.

Place and Duration of Study: Bolu Abant Izzet Baysal University Medical Faculty, Turkey; and the study was conducted between October 2017 and June 2018.

Methodology: P-wave duration and dispersions were determined on 12-lead surface ECG in 47 morbidly obese and 44 healthy weight subjects, aged between 21-40 years. Above mentioned risk factors were studied. The correlation between BMI, PD and Pmax were investigated by Pearson correlation analysis.

Results: Average body mass index (BMI) of obese and control groups were 42.3 (8.5) vs. 19.5 (1.5) (P <0.001). Maximum Pwave duration [(Pmax), 105.3 \pm 9.8 vs. 95.6 $\pm 8.5, p<0.001]$ and PD [27.6 (7.6) vs. 12.2(8.3), $\mathrm{p}<0.001]$ were statistically significantly prolonged in obese patients when compared to the normal weight group. BMI correlation with Pmax and PD ( $r=0.485$; $p<0.001$ and $r=0.620 ; p<0.001$, respectively) were significant.

Conclusion: Pmax and PD, which are potential electrocardiographic AF predictors, may increase in lone obese patients having no comorbidities.
\end{abstract}

Key Words: Electrocardiography, Arrhythmia, P-wave duration, P-wave dispersion, Severe obesity.

How to cite this article: Cosgun M, Sincer I, Inanir M, Erdal E, Mansiroglu AK, Gunes Y. P-wave Duration and Dispersion in Lone Obesity. J Coll Physicians Surg Pak 2021; 31(05):567-570.

\section{INTRODUCTION}

Obesity is associated with the developmental of cardiovascular diseases (CVDs) as an uncontrolled risk factor with a gradually increasing frequency due to the increase of atrial fibrillation (AF) in obese subjects. ${ }^{1}$ However, the potential mechanisms that increase AF risks are not precise. Obesity-related conditions like hypertension, left ventricular hypertrophy, sleep apnea and left atrial enlargement are potential risk factors for AF development. ${ }^{2,3}$ Age by itself is associated with increased AF frequency.

P-wave dispersion (PD) is one of the essential non-invasive ECG markers for assessment of AF development. ${ }^{4-7}$ Compared to normal weight subjects, PD has been shown to be prolonged in obese subjects. ${ }^{8} \mathrm{AF}$ is more frequent, and PD is prolonged in patients with hypertension, diabetes and older age, and PD is prolonged. ${ }^{7}$

Correspondence to: Dr. Mehmet Cosgun, Department of Cardiology, Bolu Abant Izzet Baysal University Hospital, Golkoy, Bolu, Turkey

E-mail:coskun44@gmail.com

Received: November 13, 2018; Revised: November 03, 2020; Accepted: December 01, 2020

DOI: https://doi.org/10.29271/jcpsp.2021.05.567
Those comorbid conditions like hypertension, diabetes, older age, atrial dilatation or ventricular hypertrophy that may be associated with both obesity and prolonged PD, were not excluded in previous studies that were searching the link between PD and obesity.

The objective of the current investigation was to study the association between PD and lone obesity, no other potential risk factors for AF development in young subjects under age of 40 years.

\section{METHODOLOGY}

This study was a cross-sectional investigation, performed at Bolu Abant Izzet Baysal University Medical Faculty between October 2017 and June 2018. The Hospital Ethics Committee approved the study protocol. The study population included 47 morbidly obese and 44 normal-weight subjects aged 21-40 years. All subjects had normal sinus rhythm. Patients taking any medications like beta-blockers or other anti-arrhythmic agents, antidepressants or having structural heart disease, heart failure, coronary artery disease, cardiac pacemaker, hypertension, atrial fibrillation, diabetes, renal failure, liver dysfunction, thyroid dysfunction, chronic obstructive pulmonary disease, anemia, electrolyte disorders, bundle branch or atrioventricular blocks on ECG, ventricular hypertrophy, atrial dilatation or diastolic dysfunction on echocardiography, were excluded from the study. 
Body mass index (BMI) was calculated by dividing the body weight by the square of the height (kilograms/meter square). $\mathrm{BMI}<25 \mathrm{~kg} / \mathrm{m}^{2}$ was accepted as normal weight, and $\mathrm{BMI} \geq 40$ $\mathrm{kg} / \mathrm{m}^{2}$ was accepted as morbid obesity (class III obesity). ${ }^{9}$ Twelve-lead ECGs were recorded after about 10 minutes of rest, with a paper speed of $25 \mathrm{~mm} / \mathrm{s}$ and amplitude of $10 \mathrm{~mm} / \mathrm{mV}$ in the supine position (Nihon Kohden Cardiofax ECG-1950 VET). Two blinded cardiologists manually measured ECGs via magnifying glass (TorQ $150 \mathrm{~mm}$ Digital Caliper LCD). P-wave duration was measured in all leads. If P-wave durations were not measurable in less than nine leads, patients were excluded. P-wave dispersion is defined as the difference between the maximum Pwave duration (Pmax) and minimum P-wave duration (Pmin). The inter- and intra-observer variations for ECG measurements were $<5 \%$.

Quantitative variables were stated as mean \pm standard deviation (SD) and median (IQR), and qualitative variables were expressed in numbers and percentages. The normality of data between groups was evaluated with Shapiro-Wilk test. The divergence among independent groups were analyzed by Student t-test in the case of normal distribution. Mann-Whitney's U-test was employed for the variables except normal distribution. Chi-square test was used for the qualitative variables. The correlation between BMI, PD and Pmax were investigated by Pearson correlation analysis. A $p$-value $<0.05$ was considered as significant. Statistical analysis was carried out by using SPSS version 15.0 software (IBM).

Table I: General characteristics and electrocardiographic findings of the groups.

\begin{tabular}{|c|c|c|c|}
\hline $\begin{array}{l}\text { Baseline } \\
\text { characteristics }\end{array}$ & $\begin{array}{l}\text { Control group } \\
(n=44)\end{array}$ & $\begin{array}{l}\text { Study group } \\
(n=47)\end{array}$ & p-value \\
\hline & \multicolumn{2}{|l|}{$\mathrm{X}^{2}$ test } & \\
\hline \multirow[t]{2}{*}{$\begin{array}{l}\text { Gender (male / } \\
\text { female) }\end{array}$} & $\begin{array}{l}12(27.3 \%) / \\
32(72.7 \%)\end{array}$ & $\begin{array}{l}11(23.4 \%) / \\
36(76.6 \%)\end{array}$ & 0.67 \\
\hline & \multicolumn{2}{|l|}{ Median (IQR) } & \\
\hline Age (years) & $32.5(6)$ & $35(8)$ & 0.27 \\
\hline BMI $\left(\mathrm{kg} / \mathrm{m}^{2}\right)$ & $19.5(1.5)$ & $42.3(8.5)$ & $<0.001$ \\
\hline \multirow[t]{2}{*}{$\mathrm{PD}, \mathrm{ms}$} & $12.2(8.3)$ & $27.6(7.6)$ & $<0.001$ \\
\hline & \multicolumn{2}{|l|}{ Mean \pm SD } & \\
\hline Pmin, ms & $82.2 \pm 8.5$ & $77.9 \pm 10.7$ & 0.04 \\
\hline Pmax, ms & $95.6 \pm 8.5$ & $105.3 \pm 9.8$ & $<0.001$ \\
\hline \multicolumn{4}{|c|}{$\begin{array}{l}\text { BMI: Body mass index, SD: Standard deviation, Pmin: Minimum P-wave } \\
\text { duration, Pmax: Maximum P-wave duration, PD: P-wave dispersion, } m \text { : } \\
\text { Millisecond. }\end{array}$} \\
\hline
\end{tabular}

\section{RESULTS}

The study population involved 47 morbidly obese subjects (11 men, 36 women) and 44 healthy-weight control subjects ( 12 men, 32 women) aged 21 to 40 years. The average age of obese patients was 35 (21 to 40 ) years. The average BMI of the morbidly obese and control groups were 42.3 (8.5) and 19.5 (1.5), $p<0.001$, respectively.

Compared to the normal weight group Pmax $(105.3 \pm 9.8 \mathrm{~ms}$ vs. $95.6 \pm 8.5 \mathrm{~ms}, p<0.001)$ was significantly longer, Pmin $(77.9 \pm$ $10.7 \mathrm{~ms} v \mathrm{vs} .82 .2 \pm 8.5 \mathrm{~ms}, \mathrm{p}=0.04$ ) was significantly lower and PD [27.6 (7.6) ms vs. 12.2 (8.3) ms, p<0.001] was significantly higher in obese patients (Table I). BMI was significantly in correlation with Pmax and PD ( $r=0.485 ; p<0.001$ and $r=0.620$; $p<0.001)$. There were no significant difference between control group and obese group regarding sex category $(27.3 \% / 72.7 \%$ vs. $23.4 \% / 76.6 \%, p=0.67)$, age ( $32.5(6)$ years vs. 35 (8) years, $\mathrm{p}=0.27$ ).

\section{DISCUSSION}

In this study, Pmax and PD were significantly increased in lone obese subjects. Obesity is a modifiable cardiovascular risk factor. ${ }^{2,3,10,11}$ Some studies have demonstrated that high blood pressure, hyperlipidemia, diabetes, and overweight/obesity are significantly intercorrelated. ${ }^{11}$ Obesity is also correlated with an elevated danger of $A F .{ }^{1-3,12-14} A$ meta-analysis including 123,249 subjects showed that obesity was linked with a $49 \%$ increased risk hazard for AF development (relative risk 1.49, $95 \% \mathrm{Cl} 1.36-1.64) .{ }^{15}$ Wang et al. reported that AF risk was increased by $4 \%$ against every one unit gain in $\mathrm{BMI}$, during a mean follow-up of 13.7 years. ${ }^{4}$ Nevertheless, the potential mechanisms explaining increased AF risk in obesity are not precise. Some co-associated conditions with obesity, such as diabetes and hypertension, also increase the likelihood of AF development. ${ }^{2}$ Left atrial dilatation and left ventricular hypertrophy are frequently present in obesity even in the absence of hypertension; and may have a role in the development of AF. Autonomic dysfunction, inflammation, and oxidative stress are among the other possible mechanisms. ${ }^{12,14}$

P-wave duration and PD, which are simple ECG parameters, have been used to predict AF risk. ${ }^{5-7}$ Increased intra-atrial conduction heterogeneity may serve as a substrate for reentry, and in consequence to $\mathrm{AF} .{ }^{12} \mathrm{PD}$ may be a simple reflection of the increased heterogeneity on surface ECG. Compared to the healthy weight subjects, Pmax and PD have been found to be prolonged in obese subjects. ${ }^{8}$ However, patients with hypertension, diabetes, older age, diastolic dysfunction, and atrial enlargement were not excluded in these studies. Kosar et al. studied 52 obese patients (age: $53 \pm 9$ years, BMI: $38.1 \pm 5.8$ ) of whom 17 were hypertensive, and 13 were diabetic. ${ }^{8}$ In the present study, the mean age of the obese patients was $34.5 \pm$ 4.5 (21-40 years), and the BMI was 45.1 \pm 5.4 . Older age, hypertension and diabetes have been associated with AF development and prolonged PD. ${ }^{7}$ Therefore, by ruling out other co-potential risk factors, this study establishes a link between PD and obesity.

Prolonged PD in morbidly obese patients is not clearly explained. Even after the adjustment for demographic and cardiovascular risk factors, severe obesity is independently associated with hypertrophy or dilatation of the heart chambers. ${ }^{13}$ The mechanisms encountered in obesity like increased neurohormonal activity, increased plasma volume and diastolic dysfunction may lead to atrial enlargement. ${ }^{3}$ Left atrial enlargement is strongly correlated with the advancement and persistence of $\mathrm{AF}^{16}{ }^{16}$ It has been shown that BMI is strongly correlated with left atrial size. ${ }^{17}$ Diastolic dysfunction has also been associated with increased PD., However, in the present study, even after the exclusion of atrial enlargement, ventric- 
ular hypertrophy and diastolic dysfunction, it is found that PD is increased in obese subjects in correlation with BMI.

Manual calculation of the ECG parameters via a magnifying lens instead of automated computer assistance is an important limitation of this study. However, some studies have reported a high accuracy for PD measurement on printed ECGs. ${ }^{18}$ Another significant constraint of this investigation is the use of small number of the study population. Longer-term and large numbered follow-up studies will be valuable for the assessment of the clinical impact of prolonged PD on the development of AF.

\section{CONCLUSION}

Here in this paper, it is found that potential electrocardiographic AF predictors, namely Pmax and PD, increase in lone obese patients with no co-associated conditions for increased AF risk. Long-term and large numbered follow-up studies might be valuable to confirm the association between increased PD and AF risk in morbidly obese patients.

\section{ETHICALAPPROVAL:}

This study was approved by Bolu Abant Izzet Baysal University Clinical Researches Ethics Committee. (Ref No. 2018/136 Date: 06.09.2018).

\section{PATIENTS' CONSENT:}

Informed consents were obtained from all individual participants included in the study.

\section{CONFLICT OF INTEREST:}

Authors declared no conflict of interest.

\section{AUTHORS' CONTRIBUTION:}

MC, IS, MI: Conception of the work, analysis and interpretation of data for the work, discussion, literature review and critically reviewed.

EE: Conception and design, acquisition of data and critically reviewed.

AKM: Drafting the study, acquisition of data and critically reviewed.

YG: Drafting the study, data acquisition, interpretation and critically reviewed.

All authors read and approved the final manuscript.

\section{REFERENCES}

1. Schnabel RB, Yin X, Gona P, Larson MG, Beiser AS, McManus DD, et al. 50 year trends in atrial fibrillation prevalence, incidence, risk factors, and mortality in the Framingham Heart Study: a cohort study. Lancet 2015; 386(9989):154-62. doi: 10.1016/S0140-6736(14)617 74-8.

2. Aune D, Sen A, Schlesinger S, Norat T, Janszky I, Romundstad $P$, et al. Body mass index, abdominal fatness, fat mass and the risk of atrial fibrillation: a systematic review and dose-response meta-analysis of prospective studies. Eur J Epidemiol 2017; 32(3):181-92. doi: 10.1007/ s10654-017-0232-4.

3. Alpert MA, Omran J, Bostick BP. Effects of Obesity on
Cardiovascular Hemodynamics, Cardiac Morphology, and Ventricular Function. Curr Obes Rep 2016; 5(4):424-34. doi: 10.1007/s13679-016-0235-6.

4. Wang TJ, Parise H, Levy D, D'Agostino RB, Sr, Wolf PA, Vasan RS, et al. Obesity and the risk of new-onset atrial fibrillation. Jama 2004; 292(20):2471-7. doi: 10.1001/ jama.292.20.2471.

5. Perez-Riera AR, de Abreu LC, Barbosa-Barros R, Grindler J, Fernandes-Cardoso A, Baranchuk A. P-wave dispersion: An update. Indian Pacing Electrophysiol J 2016; 16(4): 126-33. doi: 10.1016/j.ipej.2016.10.002.

6. Okutucu S, Aytemir K, Oto A. P-wave dispersion: What we know till now? JRSM Cardiovasc Dis 2016; 5:2048004 016639443. doi: 10.1177/2048004016639443.

7. Nielsen JB, Kuhl JT, Pietersen A, Graff C, Lind B, Struijk JJ, et al. P-wave duration and the risk of atrial fibrillation: Results from the copenhagen ECG study. Heart Rhythm 2015; 12(9):1887-95. doi: 10.1016/j.hrthm.2015.04.026.

8. Kosar F, Aksoy Y, Ari F, Keskin L, Sahin I. P-wave duration and dispersion in obese subjects. Ann Noninvasive Electrocardiol 2008; 13(1):3-7. doi: 10.1111/j.1542474X.2007.00194.x.

9. Organization WH. Obesity: Preventing and managing the global epidemic: World Health Organization; 2000.

10. Firdous $\mathrm{S}$. Correlation of CRP, fasting serum triglycerides and obesity as cardiovascular risk factors. J Coll Physicians Surg Pak 2014; 24(5):308-13.

11. Bhupathiraju SN, Hu FB. Epidemiology of Obesity and Diabetes and Their Cardiovascular Complications. Circ Res 2016; 118(11):1723-35. doi: 10.1161/CIRCRESAHA. 115. 306825.

12. Ebong IA, Goff DC, Jr., Rodriguez CJ, Chen H, Bertoni AG. Mechanisms of heart failure in obesity. Obes Res Clin Pract 2014; 8(6):e540-8. doi: 10.1016/j.orcp.2013. 12.005.

13. Goudis CA, Korantzopoulos P, Ntalas IV, Kallergis EM, Ketikoglou DG. Obesity and atrial fibrillation: A comprehensive review of the pathophysiological mechanisms and links. J Cardiol 2015; 66(5):361-9. doi: 10.1016/ j.jjcc.2015.04.002.

14. Nalliah CJ, Sanders P, Kottkamp H, Kalman JM. The role of obesity in atrial fibrillation. Eur Heart J 2016; 37(20): 1565- 72. doi: 10.1093/eurheartj/ehv486.

15. Wanahita N, Messerli FH, Bangalore S, Gami AS, Somers VK, Steinberg JS. Atrial fibrillation and obesity--results of a meta-analysis. Am Heart J 2008; 155(2):310-5. doi: 10.1016/j.ahj.2007.10.004.

16. Padfield GJ, Steinberg C, Swampillai J, Qian H, Connolly SJ, Dorian $\mathrm{P}$, et al. Progression of paroxysmal to persistent atrial fibrillation: 10-year follow-up in the Canadian Registry of Atrial Fibrillation. Heart Rhythm 2017; 14(6):801-7. doi: 10.1016/j.hrthm.2017.01.038.

17. Huang G, Parikh PB, Malhotra A, Gruberg L, Kort S. Relation of Body Mass Index and Gender to Left Atrial Size and Atrial Fibrillation. Am J Cardiol 2017; 120(2): 218-22. doi: 10.1016/j.amjcard.2017.04.013. 
18. Pall A, Czifra A, Sebestyen V, Becs G, Kun C, Balla J, et al. Hemodiafiltration and hemodialysis differently affect $P$ wave duration and dispersion on the surface electrocardiogram. Int Urol Nephrol 2016; 48(2):271-7. doi: 10.1007/s11255-015-1144-4. 